\title{
Traffic:
}

\section{Authorizing Airspace, Appifying Governance}

\author{
Marcel LaFlamme \\ Ludwig Boltzmann Gesellschaft \\ (iD) https://orcid.org/0000-0002-7489-4233
}

Author's version of chapter in Voluminous States: Sovereignty, Materiality, and the Territorial Imagination, edited by Franck Billé (Durham, NC: Duke University Press, 2020), 91-102. https://doi.org/10.1215/9781478012061-007. C 2020 Duke University Press. 


\title{
Traffic:
}

\section{Authorizing Airspace, Appifying Governance}

\author{
by Marcel LaFlamme
}

Pete spotted the brown car first, parked along an access road near the section he was spraying for a farmer west of Fargo. Half an hour later, it was on the move, and a gray car pulled up beside it at the other end of the field. The two drivers stood and talked for a while, and Pete wondered: "What are they doing out here?" He kept working his way across the section, flying mile-long passes from north to south and then back again, applying an herbicide to the crops below. Four hours in, he dove down for another pass and suddenly saw a drone beneath him and behind his wing, not 250 feet away. His pulse was pounding as he leveled out and then climbed to a safe distance, reaching for his radio to figure out what the hell was going on. Getting no response, Pete headed back to the hangar, where his boss was incensed. A propeller strike would have caused upward of $\$ 80,000$ in damage, plus downtime during a growing season that lasts just a few months on the Upper Great Plains. But Pete's first thought was for his wife and kids: life as an aerial applicator is dangerous enough without a carbon-fiber camera coming through your windshield.

It was the summer of 2014 when Pete recounted this story to me, just as I was wrapping up a year of research on efforts to position the state of North Dakota as a center of the unmanned aviation industry. Technically, I knew, drones like the one that Pete encountered were not supposed to be flying outside of strictly regulated test sites. But it was no secret that companies had sprung up to collect crop imagery in this way, and the farmer whose field Pete was spraying had evidently hired one of them. It was a delicate situation: the farmer was a good customer, and so Pete's boss was reluctant to stir up trouble by reporting the incident to the authorities. Yet norms had been violated, and the prospect of a midair collision was one that could not be taken lightly. "If another pilot got that close to me without talking about it, I'm probably going to get ugly with him," Pete vented. Ordinarily, aerial applicators used the radio to sort out conflicting flight paths; failing that, they could at least acknowledge each other's presence by emitting puffs of smoke from their wings. But the drone was associated with a new kind of airspace user, one who did not necessarily think to take other traffic into account. "To a ground operator not used to being around airplanes," Pete reflected, "what's distance? A half mile feels like forever on a gravel road." 
By the mid-twentieth century, promoting the safe separation of air traffic was understood to be part of how sovereign states governed in three dimensions. The transfer of radar technology from military to civilian settings brought vast expanses of airspace under positive control. Far from all-seeing, though, these technical systems supported processes of cooperation and coordination among skilled air traffic controllers. ${ }^{1}$ In this chapter, I show how the maturation and proliferation of unmanned aircraft or drones has posed challenges to existing regimes of traffic management and given rise to new modes of volumetric sovereignty. In particular, I highlight the growing importance of what Keller Easterling has called extrastatecraft, infrastructural orchestrations that are conducted "outside of, in addition to, and sometimes even in partnership with statecraft." 2 Admittedly, the extent to which air traffic control remains the direct responsibility of the U.S. government is exceptional in a global context, as new public management paradigms have prompted many countries to transfer system management to various corporate entities. ${ }^{3}$ Still, I argue that the particular form of marketization that is underway in the United States reveals how new technologies are transforming the way in which sovereignty is governed across borders as well as within them.

It is no accident that the smartphone is becoming a key site for the governance of drone mobilities, since both consumer technologies followed on the miniaturization of electromechanical systems and advances in microprocessor design during the first decade of the twenty-first century. As smartphones became increasingly ubiquitous, software developers looked to them as a platform for the delivery of digital tools called apps that would accomplish specific tasks in a richly visual way. Media scholars Jeremy Wade Morris and Sarah Murray insightfully frame apps as "mundane software," in the double sense of being ordinary and readily accessible out in the world. ${ }^{4}$ In what follows, I consider an initiative to provide U.S. drone operators with expedited access to controlled airspace through the mediation of app-based service providers. Insofar as this program manages to automate the process of airspace authorization, it can be approached in the context of concerns about "the way in which digital data is troubling and reconstituting expertise." 5 Yet because the provision of traffic management expertise is bound up with specific political formations, the program also opens out onto broader questions about how and to whom sovereign states grant the authority to govern the sky. 


\section{At the Data Exchange}

In 2016, two full years after Pete's near-miss, the Federal Aviation Administration (FAA) finally issued a set of regulations around the use of drones for commercial purposes. Under these regulations, a drone weighing less than fifty-five pounds could be flown by a licensed operator up to a maximum altitude of four hundred feet. Yet this permission was not unconditional: if the flight was going to take place at night, over people, or beyond the operator's line of sight, then a waiver request would need to be filed at least ninety days in advance. Flights that would do none of those things but would take place within five miles of an airport could go through a separate authorization process, which was supposed to be streamlined. But an audit found that the office at the FAA charged with reviewing these requests was swamped, even after hiring additional contractors: wait times for a routine airspace authorization hovered around six weeks, frustrating business owners who were trying to play by the rules but who found themselves outmaneuvered by less scrupulous competitors. With almost four thousand requests coming in each month, the agency was under pressure to make the process more efficient or risk losing control of it altogether.

Enter the Low Altitude Authorization and Notification Capability (LAANC), which was introduced on a trial basis in November 2017 and rolled out nationwide during 2018. Rather than having a human analyst review authorization requests on a case-by-case basis, LAANC converts these requests into a machine-readable format and matches them against a repository of airport facility maps and other data sources; if no conflicts are detected, then an authorization is transmitted to the requester in near real time. Notably, the concept of operations for LAANC stipulates that the program "will not involve or result in a government acquisition of software programs or systems." " Instead, LAANC operates as a public-private partnership between the FAA and socalled service suppliers, technology companies that build (and seek to monetize) the software by which drone operators make airspace requests. This division of labor relieves the FAA of the responsibility for developing its own software internally, and it aligns with the agency's stated aim to "foster a free market environment" for drone service providers.

It would be easy to interpret this arrangement in terms of a broader deregulation agenda ushered in by the election of Donald Trump in 2016. Trump did initially endorse a plan to privatize the U.S. air traffic control system (before backing down in the face of opposition from his rural base), and his secretary of transportation, Elaine Chao, clearly telegraphed her desire to remove 
government roadblocks and empower the drone industry during her Senate confirmation hearings. But the vision of coordination among public and private entities behind LAANC actually has its roots in the Obama administration, most proximately in a traffic management system concept being developed by NASA but more fundamentally, I argue, in an embrace of open civic data. ${ }^{8}$ In this paradigm, public agencies make the data that they collect and produce freely available on a nonexclusive basis, and then private-sector "infomediaries" develop products and services that build on this data and add value to it. ${ }^{9}$ For LAANC service suppliers, access to an FAA-operated data exchange is predicated on compliance with agency standards and interoperability with other stakeholders. The model is thus one of virtuous, market-based competition on the basis of a level playing field constituted by public data. Yet, as these emerging techniques of extrastatecraft are codified, the question of their relationship to state sovereignty is often elided. Hence, the LAANC concept of operations insists that the automatic authorization process "is not a delegation of authority by the FAA to the [service supplier. Service suppliers] assist operators to identify preapproved authorizations as defined by regulations in collaboration with the FAA."10

It is, I suggest, in this gap between delegation and collaboration that new modes of volumetric sovereignty are starting to coalesce. In the next section, I seek to chart their contours by offering a walkthrough of one app being used by U.S. drone operators to request airspace authorizations, drawing on a methodology elaborated by scholars of new media. ${ }^{11}$ The researcherinitiated walkthrough, while no substitute for ethnographic research with app users, does offer a structured look at a complex digital object demanding both technical and cultural analysis. The walkthrough tacks between direct observation and manipulation of the app's interface and a document-based assessment of the app's environment of expected use. In the context of LAANC, I take this environment to include the data sources and flows that give the app its dynamism, as well as those conditions that elude datafication and inscribe the app within a broader ecology of aviation media. ${ }^{12}$ To trace these admittedly arcane processes is to grasp how both sky and state are being remade by digital technologies and their remainders. 


\section{On (and Off) the Flight Deck}

Named for the windy stretch of beach where the Wright brothers first flew in 1903, Kittyhawk is one of the leading apps for planning, monitoring, and managing data associated with drone flights. Created in 2015 as a tool for flight logging, the app rapidly evolved new features and today its San Francisco-based developers describe it as a "single system of record for drone operations." 13 In published interviews, Kittyhawk cofounders Joshua Ziering and Jon Hegranes describe their target user as a business that had previously hired external service providers but now wants to move its drone program in-house. Outside expertise is no longer necessary, they contend, given the development of what Kittyhawk's website calls "software anyone can use."14 Indeed, Ziering provocatively argues that "good software is going to abstract away the notion of the drone," decentering the airframe and the details of its configuration in favor of a focus on value delivered through a dematerialized stream of data-driven insight. ${ }^{15}$

I downloaded Kittyhawk from the App Store, where it was categorized under "Business," to my iPhone XR in December 2018. The fact that I was able to do so at all distinguishes Kittyhawk from competitor products like the Verizon-owned Skyward, which cannot be found in consumerfacing app stores and must be demoed by a sales representative. Kittyhawk's discoverability on these platforms reflects its present commitment to continue offering a free product to individual end users, an important segment of its original user base. As the app took me through the process of creating an account, I was never asked to choose between free and paid plans: it was only upon trying to access specific features like streaming media that I was invited to "unlock this powerful feature with a Kittyhawk Enterprise account." Significantly, this requester directed me to a contact form that did not include any information about pricing, although as recently as September 2018 a pricing page on the Kittyhawk website offered a small business plan for \$24 per month per user as well as a "solopreneur" option. The collapse of these categories into a single "Enterprise" tier indexes a strategic decision to focus on larger, more lucrative clients, but it also allows Kittyhawk to set pricing without being constrained by a uniform, publicly accessible rate schedule.

Upon opening the Kittyhawk app, the default view is divided between a map of the user's current location and two ribbon-style control panels, labeled "Flight Conditions" and "Flight Deck." The former panel, which is dynamically updated, shows environmental conditions including wind speed, visibility, and cloud cover, underlining values of possible concern for a drone operator in 
either yellow or red. The latter allows the user to scroll through an array of tools for completing checklists, reviewing advisory notices, and even controlling a drone from within the app. Thus, requesting airspace authorizations through LAANC is only one feature of Kittyhawk, although it is seen as a critical one. When Kittyhawk was not invited to participate in the evaluation phase of LAANC, Ziering groused that "giving certain companies months of exclusive access to a precious resource is at best anticompetitive." 16 Within months, though, Kittyhawk had lined up a partnership with the Boeing subsidiary Jeppesen, which came with an infusion of capital and the use of Jeppesen's electronic navigation charts but also with access to the LAANC data exchange. By forging this alliance, Kittyhawk managed to reroute sovereign power around what it viewed as an arbitrary blockage and to stay apace of its competitors in the scramble for market position. In October 2018, the FAA announced the selection of Kittyhawk as a LAANC service supplier in its own right; a video released by the company following the announcement hailed LAANC as a "major innovation" and noted that "we're really excited to offer it."17

Before requesting an airspace authorization through the Kittyhawk app, users must complete a "LAANC Readiness" checklist. Users are asked to supply their Part 107 Certificate number, which drone operators receive upon completion of a licensing process that includes a written exam. Users also need to have at least one aircraft registered with the FAA and entered into Kittyhawk's "Assets" module. Yet the most prosaic part of the checklist may, in fact, say the most about the tension between state and market that is at the heart of LAANC. Without any explanation, users are asked to verify the phone number that they supplied upon initially creating an account with Kittyhawk: a six-digit verification code is sent to the user via text message, and once the code is typed into the app the status of the user's phone number changes to "Verified." Phone verification is a fairly common security measure used by app developers, but it carries a particular significance for LAANC service suppliers. The LAANC concept of operations specifies that "for the purposes of communication between air traffic control and operators during flight events, a valid U.S. phone number . . . that can be used to reach the operator at any time during flight operations will be required for all notifications and authorizations." 18 Thus, even as app developers like Kittyhawk aspire to create "a single system of record for drone operations," the FAA reserves the right to communicate with operators outside of the app through the residual medium of telephony. ${ }^{19}$ By giving service suppliers the task of collecting and validating phone numbers, the FAA at once absolves itself of the responsibility to manage personally identifiable information and retains the 
prerogative to disintermediate the service supplier, reasserting the dyad of sovereign and subject with an old-fashioned voice call.

Once the "LAANC Readiness" checklist is complete, Kittyhawk users can request an airspace authorization from a menu at the bottom of the app's default view. On one screen, the user enters the date, time, and duration of the requested authorization. Next, the app loads a map on which the user can define their intended flight area: a shaded circle indicates the five-mile radius of controlled airspace around larger airports, while yellow and green gridlines show the altitudes up to which drone operations have been preapproved by air traffic controllers at those airports. The Kittyhawk user drags a pin marked with the company's cloverleaf logo to the relevant area of the map and uses a drawing tool to mark off the desired airspace. Finally, the user ticks a series of checkboxes affirming their compliance with FAA regulations and can then submit their authorization request. Kittyhawk transmits a record of the request to the FAA, which will subsequently share it with air traffic controllers at the affected airport. But what actually triggers the authorization is the query that Kittyhawk sends to the LAANC data exchange, confirming that the request does not conflict with any existing restrictions or advisories. The authorization message that Kittyhawk sends to the user on the FAA's behalf is, in a sense, a mere trace of that query: an instantiation of volumetric sovereignty dynamically generated by a conversation between machines. 


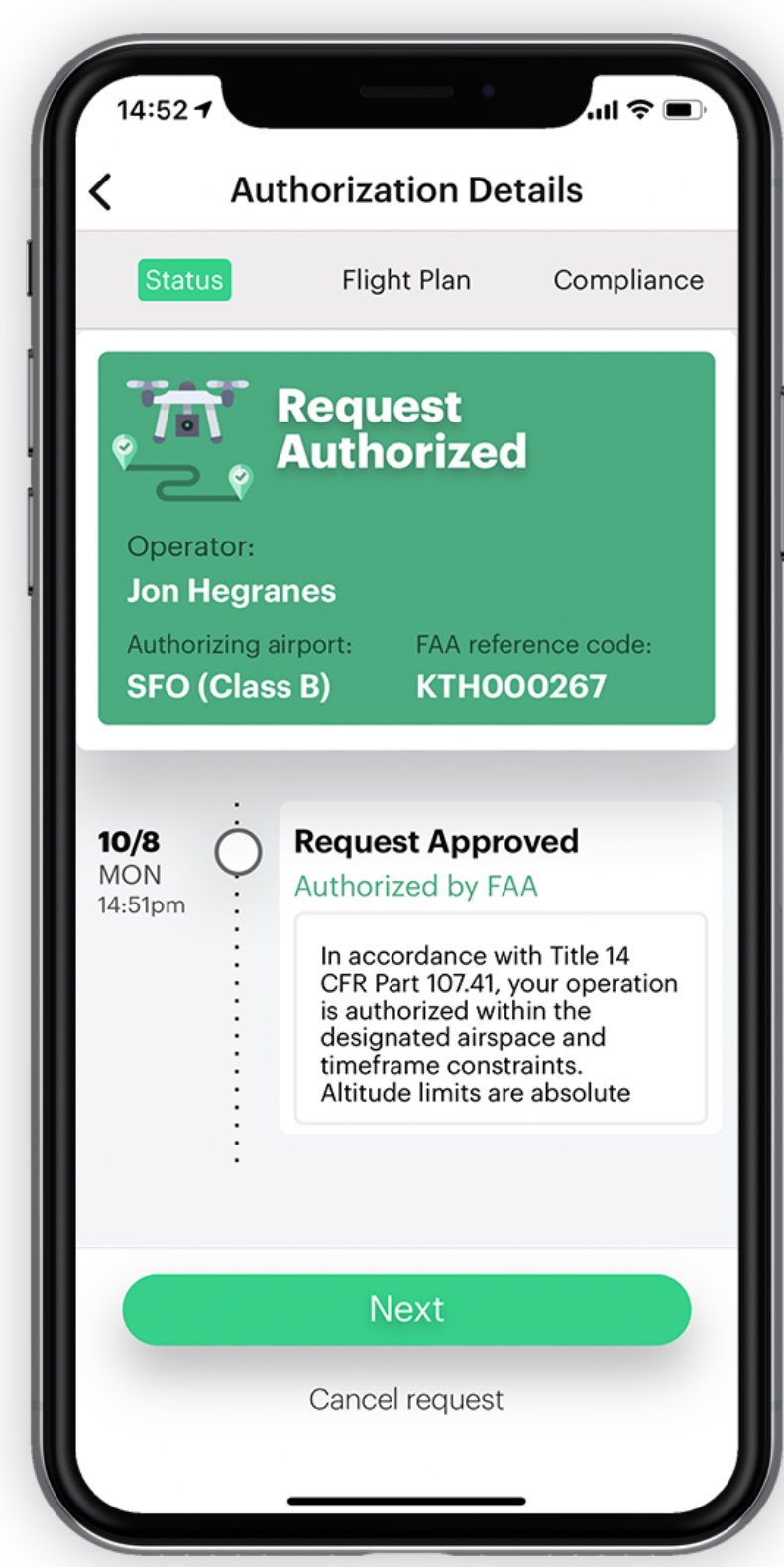

Figure 1. A LAANC authorization in the Kittyhawk app. Image courtesy of Kittyhawk.

What are we to make of this curious document? Aesthetically speaking, its legal standing would seem to be at odds with its bright green background and the cartoonish drone hovering in the upper left corner; the FAA logo and other branding elements are nowhere in evidence. The message does include an FAA reference code, which includes the initials "KTH" to designate the service supplier with which the authorization is associated. Kittyhawk's "Assets" module includes 
a section where users can review all of their approved authorizations, as well as requests that have been submitted to the FAA for what the app terms "further coordination." If an airspace request is otherwise eligible for authorization but exceeds preapproved altitude limits, it can be directed to the relevant air traffic controller for manual review, which may involve the requester providing additional information. Here, the seductive seamlessness promised by LAANC breaks down: manual review can take thirty days or more, and a request under review is automatically canceled if a decision has not been reached twenty-hour hours before the planned operation. Giving users a way to submit requests for further coordination arguably makes apps like Kittyhawk more versatile, allowing them to keep users inside the app rather than sending them beyond its confines. Yet this capability is notably absent from Kittyhawk's upbeat promotional video about LAANC, because the very need for its existence discloses the human labor and judgment that — at least for nowstand in the way of a fully automated airspace.

\section{Conclusion}

In November 2018, one year after LAANC's soft launch, the FAA put out a press release noting that the rollout has "exceeded all of the program's original objectives," with more than fifty thousand requests processed. ${ }^{20}$ This is a considerable accomplishment, with concrete benefits for both drone operators and overburdened government workers, and I do not mean to diminish it. In closing, however, I want to draw out the implications of those objectives, both for other airspace users and for how social researchers think about volumetric sovereignty. It is not clear, for instance, that LAANC has made flying any safer for pilots like my informant Pete, whose close encounter with a drone opened this chapter. Since the airspace where Pete was flying-like most of the airspace over rural North Dakota - was uncontrolled, the drone's operator would have been under no obligation to request an authorization. Now, LAANC service suppliers know that the demand for information about drone operations extends beyond agents of the state, and many are participating in the development of a new standard called Remote ID, which will allow operators to share flight data with interested observers near their location. Like LAANC, Remote ID avoids betting on any one service supplier by emphasizing interoperability. For the sector as a whole, though, such a platform represents a bid for what media scholars have called infrastructuralization, with its adoption becoming a de facto requirement for accessing airspace at all. ${ }^{21}$ 
There is extrastatecraft afoot, and yet let us not count more conventional logics of sovereignty out too quickly. As Keller Easterling observes, new dispensations of governance under contemporary capitalism tend to "strengthen rather than diminish the power of the state."22 Consider the wariness around DJI, the Chinese manufacturer that today comprises 74 percent of the commercial drone market. In 2017, the U.S. Army directed units in the field to stop using DJI drones due to concerns about "cyber vulnerabilities," while an intelligence bulletin from U.S. Immigration and Customs Enforcement alleged that DJI was "likely providing U.S. critical infrastructure and law enforcement data to [the] Chinese government." ${ }^{23}$ Amid these concerns about data security, tinged as they were with geopolitical intrigue, Kittyhawk briefly positioned itself as an American-made solution. "If you are using [the app distributed with DJI drones]," Ziering told Aviation Today, "it's talking to China and that is making a lot of people nervous. We have customers that are specifically mandated to be domestically stored, and [Kittyhawk] offers them the ability to fly their DJI stuff and not have their data go to China." 24 To counter this narrative, DJI added a privacy mode to its app and patched some widely publicized security flaws. One indication of the success of its damage control was that, in October 2018, DJI was approved as a LAANC service supplier - on the very same day as Kittyhawk. As companies like these jockey for position, what seems clear is that the evolving project of governance in three dimensions will unfold against the backdrop of bordering processes driven by both state and nonstate actors.

Regardless of where the seat of volumetric sovereignty is understood to reside, the extent of its ambition also demands to be interrogated. For is it, in the end, hubris to imagine establishing positive control over every object that intrudes into the firmament? The age of the drone has brought this question to the fore by redefining what counts as navigable (and thus governable) airspace down into the human lifeworld. I still recall sitting on an open-air patio by the Red River one summer evening in North Dakota, swapping notes with a young drone entrepreneur. Setting his beer down for a moment, he raised his arms above his head and swung his hands in circles: "This is the National Airspace System," I remember him telling me, his tone incredulous. The representational technologies and regimes of expertise that regulated air traffic in the twentieth century are stymied by the task of securing the volume of air just above our heads that evening. But if that volume does need securing (and it may), then the software charged with doing so stands to challenge exceptionalisms around both the state and the human as the locus of sovereign decision. 


\section{Notes}

${ }^{1}$ John A. Hughes, David Randall, and Dan Shapiro, "Faltering from Ethnography to Design," Proceedings of the 1992 ACM Conference on Computer-Supported Cooperative Work (1992): $115-22$.

${ }^{2}$ Keller Easterling, Extrastatecraft: The Power of Infrastructure Space (New York: Verso, 2014), 6.

${ }^{3}$ Rui Neiva, Institutional Reform of Air Navigation Service Providers: A Historical and Economic Perspective (Northampton, MA: Edward Elgar, 2015).

${ }^{4}$ Jeremy Wade Morris and Sarah Murray, "Introduction," in Appified: Culture in the Age of Apps, ed. Jeremy Wade Morris and Sarah Murray (Ann Arbor: University of Michigan Press, 2018), 9.

${ }^{5}$ Hannah Knox and Dawn Nafus, "Introduction: Ethnography for a Data-Saturated World," in Ethnography for a Data Saturated World, ed. Hannah Knox and Dawn Nafus (Manchester, UK: Manchester University Press, 2018), 3-4.

${ }^{6}$ FAA, "Low Altitude Authorization and Notification Capability (LAANC) Concept of Operations," version 1.1, May 12, 2017, 2, https://www.faa.gov/uas/programs_partnerships/ data_exchange/laanc_for_industry/media/laanc_concept_of_operations.pdf.

${ }^{7}$ FAA, "Low Altitude Authorization," 1.

${ }^{8}$ Parimal Kopardekar, Joseph Rios, Thomas Prevot, Marcus Johnson, Jaewoo Jung, and John E. Robinson III, "Unmanned Aircraft System Traffic Management (UTM) Concept of Operations" (paper presented at the American Institute of Aeronautics and Astronautics Aviation Technology, Integration, and Operations Conference, Washington, DC, June 13-17, 2016).

${ }^{9}$ Marijn Janssen and Anneke Zuiderwijk, "Infomediary Business Models for Connecting Open Data Providers and Users," Social Science Computer Review 32 (2014): 694-711.

${ }^{10}$ FAA, "Low Altitude Authorization," 8.

${ }^{11}$ Ben Light, Jean Burgess, and Stefanie Duguay, "The Walkthrough Method: An Approach to the Study of Apps," New Media and Society 20 (2018): 881-900.

${ }^{12}$ Marcel LaFlamme, "A Sky Full of Signal: Aviation Media in the Age of the Drone," Media, Culture and Society 40 (2018): 689-706.

${ }^{13}$ Kittyhawk website, "Enterprise," accessed December 9, 2019, https://kittyhawk.io/enterprise.

${ }^{14}$ Kittyhawk website, "Product," accessed December 9, 2019, https://kittyhawk.io/product.

${ }^{15}$ Joshua Ziering, "Abstraction as a Service Comes to Drones," Kittyhawk blog, January 3, 2018, accessed February 11, 2019, https://kittyhawk.io/blog/abstraction-as-a-service-comes-todrones.

${ }^{16}$ Joshua Ziering, “LAANC Fact Check: Can You Hear Me Now?," LinkedIn post, October 23, 2017, accessed February 11, 2019, https://www.linkedin.com/pulse/laanc-fact-check-can-youhear-me-now-joshua-ziering.

${ }^{17}$ Kittyhawk, "Overview of LAANC," YouTube video, October 15, 2018, https://www. youtube.com/watch? $=\mathrm{v}=\mathrm{gO} Z \mathrm{ZuSYUjN} 4$. 
${ }^{18}$ FAA, "Low Altitude Authorization," 9.

${ }^{19}$ Charles R. Acland, ed., Residual Media (Minneapolis: University of Minnesota Press, 2006).

${ }^{20}$ FAA, "More than Fifty Thousand LAANC Authorizations Processed," News, November 19, 2018, https://www.faa.gov/news/updates/?newsId=92273.

${ }^{21}$ Jean-Christophe Plantin, Carl Lagoze, Paul N. Edwards, and Christian Sandvig, "Infrastructure Studies Meet Platform Studies in the Age of Google and Facebook," New Media and Society 20 (2018): 293-310. See also Carolin Gerlitz, Anne Helmond, David B. Nieborg, and Fernando N. van der Vlist, "Apps and Infrastructures: A Research Agenda," Computational Culture 7 (2019), http://computationalculture.net/apps-and-infrastructures-a-research-agenda.

22 Easterling, Extrastatecraft, 49, fn63.

${ }^{23}$ Department of the Army, Memorandum, "Discontinue Use of Dajiang Innovation (DJI) Corporation Unmanned Aircraft Systems," August 2, 2017; U.S. Immigration and Customs Enforcement, SAC Intelligence Program Los Angeles, Intelligence Bulletin, "Dajiang Innovations (DJI) Likely Providing U.S. Critical Infrastructure and Law Enforcement Data to Chinese Government," August 9, 2017.

24 “Kittyhawk Drone Flight Deck Keeps DJI GO Data Domestically Stored," Aviation Today, May 17, 2017, https:/www.aviationtoday.com/2017/05/17/ kittyhawk-drone-flight-deck-keepsdji-go-data-domestically-stored. 